\title{
Unstable Pathologic Vertebral Fractures in Multiple Myeloma : Propensity Score Matched Cohort Study between Reconstructive Surgery with Adjuvant Radiotherapy and Radiotherapy Alone
}

\author{
Hyung-Youl Park, ${ }^{1}$ Young-Hoon Kim, ${ }^{2}$ Joo-Hyun Ahn, ${ }^{3}$ Kee-Yong Ha, ${ }^{4}$ Sang-Il Kim, ${ }^{2}$ Jae-Woong Jung ${ }^{1}$ \\ Department of Orthopaedic Surgery, Eunpyeong St. Mary's Hospital, College of Medicine, The Catholic University of Korea, Seoul, Korea \\ Department of Orthopaedic Surgery, ${ }^{2}$ Seoul St. Mary's Hospital, College of Medicine, The Catholic University of Korea, Seoul, Korea \\ Department of Orthopaedic Surgery, ${ }^{3}$ Mediplex Sejong Hospital, Incheon, Korea \\ Department of Orthopedic Surgery, ${ }^{4}$ Kyung Hee University Hospital at Gangdong, Seoul, Korea
}

Objective : Although radiotherapy (RT) is recommended for multiple myeloma (MM) involving spine, the treatment of choice between reconstructive surgery with RT and RT alone for pathologic vertebral fractures (PVFs) associated with structural instability or neurologic compromises remains controversial. The purpose of this study was to evaluate the clinical efficacies of reconstructive surgery with adjuvant RT for treatment of MM with PVFs by comparing with matched cohorts treated with RT alone.

Methods : Twenty-eight patients underwent reconstructive surgery followed by RT between 2008 and 2015 in a single institution, for management of PVFs associated with structural instability of the spine and/or neurologic compromises (group I). Twentyeight patients were treated with RT alone (group II) after propensity score matching in a 1-to-1 format based on instability of the spine, as well as age and performance. Clinical outcomes including the overall survival rates, duration of independent ambulation, neurological status, and numeric rating scale (NRS) for back pain were compared.

Results : Clinical and radiological features before treatment were similar in both groups. The median survival period was similar between the two groups. However, the mean duration of independent ambulation was significantly longer in group I (88.8 months; $95 \%$ confidence interval $[\mathrm{Cl}], 66.0-111.5)$ than in group II (39.4 months; $95 \% \mathrm{Cl}, 25.2-53.6)$ (log rank test; $p=0.022)$. Deterioration of Frankel grade ( $21.4 \%$ vs. $60.7 \%, p=0.024)$ and NRS for back pain $(2.7 \pm 2.2$ vs. $5.0 \pm 2.7, p=0.000)$ at the last follow-up were higher in the group II. Treatment-related complications were similar in both groups.

Conclusion : In patients with unstable PVFs due to MM, reconstructive surgery may yield superior clinical outcomes compared with RT alone in maintaining independent ambulation and neurological status, as well as pain control despite similar median survival and complications.

Key Words : Spine $\cdot$ Multiple myeloma $\cdot$ Surgery $\cdot$ Radiotherapy.

- Received : August 2, $2021 \cdot$ Accepted : August 12, 2021

- Address for reprints : Young-Hoon Kim

Department of Orthopaedic Surgery, Seoul St. Mary's Hospital, College of Medicine, The Catholic University of Korea, 222 Banpo-daero, Seocho-gu, Seoul 06591, Korea Tel : +82-2-2258-2837, Fax : +82-2-535-9834, E-mail : boscoa@catholic.ac.kr, ORCID : https://orcid.org/0000-0003-1237-4600 


\section{INTRODUCTION}

Multiple myeloma (MM) is a hematological malignancy in which malignant plasma cells accumulate in the bone marrow and secrete monoclonal immunoglobulin ${ }^{9)}$. MM is unique among hematological malignancies involving musculoskeletal system at the time of initial diagnosis ${ }^{9,32}$. Spine is the most afflicted skeletal lesion and pathologic vertebral fractures (PVFs) were diagnosed radiologically in $48 \%$ of 1027 patients with $\mathrm{MM}^{14,17,18)}$.

Although chemotherapy is the mainstay of treatment for $\mathrm{MM}$, radiotherapy (RT) or surgery may play an important role in cases of intractable back pain or spinal cord compression resulting from spine involvement ${ }^{2,4,13,18,22,31,34)}$. First, RT rather than surgical treatment is recommended because of acceptable radiosensitivity, especially for patients with MM in poor health condition such as renal failure or anemia ${ }^{3,12,16,18,20,22,27,31,32,34)}$. Even in PVFs, cement augmentation is considered as minimally invasive and effective treatment, because reconstructive surgery is associated with a higher rate of complications ${ }^{2-4,13,18,20,22,27,31)}$.

Reconstructive surgical interventions are needed in a few cases with structural instability or neurologic compromis$\mathrm{es}^{8,15,33)}$. However, few studies have investigated the role of reconstructive surgery due to the rarity of disease ${ }^{3,27,31,34)}$. Therefore, the purpose of this study is to assess the clinical efficacy of reconstructive surgery with adjuvant RT for PVFs associated with significant structural instability or neurologic compromises in patients with MM, by comparing with matched cohorts treated with RT alone.

\section{MATERIALS AND METHODS}

This study was approved by the Institutional Review Board of Seoul St. Mary's Hospital, College of Medicine, The Catholic University of Korea (approval No. KC17DDDE0117). A clinical and radiological database (2008 to 2015) was retrospectively reviewed at a single tertiary institution.

\section{Study population}

Twenty-eight patients who underwent reconstructive surgery followed by RT for PVFs with structural instability or neurologic compromises were included as group I (surgery+RT group). Vertebral fractures with significant structural instabili- ty and/or progressive deterioration of neurologic deficits were indicated for reconstructive surgery. Structural instability of the spine was assessed with radiologic findings such as location and spinal alignment, collapse and osteolysis of vertebral body or spine instability neoplastic score (SINS). SINS value higher than 7 could be considered as significant instability. In the same period (2008 to 2015), a total of 257 patients were treated with RT for MM involving spine. PVFs were found in 119 patients (46.3\%) and 28 patients were manually matched 1 to 1 using propensity score and included as group II (RT alone group). For propensity score matching, age, Eastern Cooperative Oncology Group-performance status (ECOG-PS) and SINS to evaluate spinal instability were used after multivariate regression analysis (Fig. 1). Patients without PVFs and patients with concurrent pelvis or hip joint involvement affecting independent ambulation were excluded.

\section{Evaluation and treatment}

All patients were diagnosed with MM via bone marrow biopsy histologically and or treated with standard chemotherapies. Spine involvement was confirmed clinically based on symptoms including back pain and neurologic deficit, and radiologically via plain radiography, computed tomography (CT), magnetic resonance imaging (MRI) or positron-emitting tomography-computed tomography.

Medical treatments including chemotherapy regimens and peripheral blood stem cell transplantation (PBSCT) were determined based on same criteria in both groups. RT was administered to all patients using the TomoTherapy ${ }^{\circledR} \mathrm{Hi}^{-A R T^{\circledR}}$ Treatment System (Accuray ${ }^{\circledR}$, Sunnyvale, CA, USA) based on CT simulation before the treatment. The median $20 \mathrm{~Gy}$ (range, 15-45) was delivered in 10 fractions during 2 weeks, and the total dose was set according to patient's functional status.

The surgical indications included spinal instability confirmed with definite radiologic findings or SINS greater than 6 , and/or neurologic impairment due to cord compres$\operatorname{sion}^{2,10,11)}$. However, decision for reconstructive surgeries was determined via a multidisciplinary approach by a team of orthopedic surgeons, hemato-oncologists, and radiation oncologists. Above all, permissible patients' conditions without requiring urgent or essential medical treatments to sustain life were mandatory for reconstructive surgery. As the objective of reconstructive surgery was to maintain the structure of the 


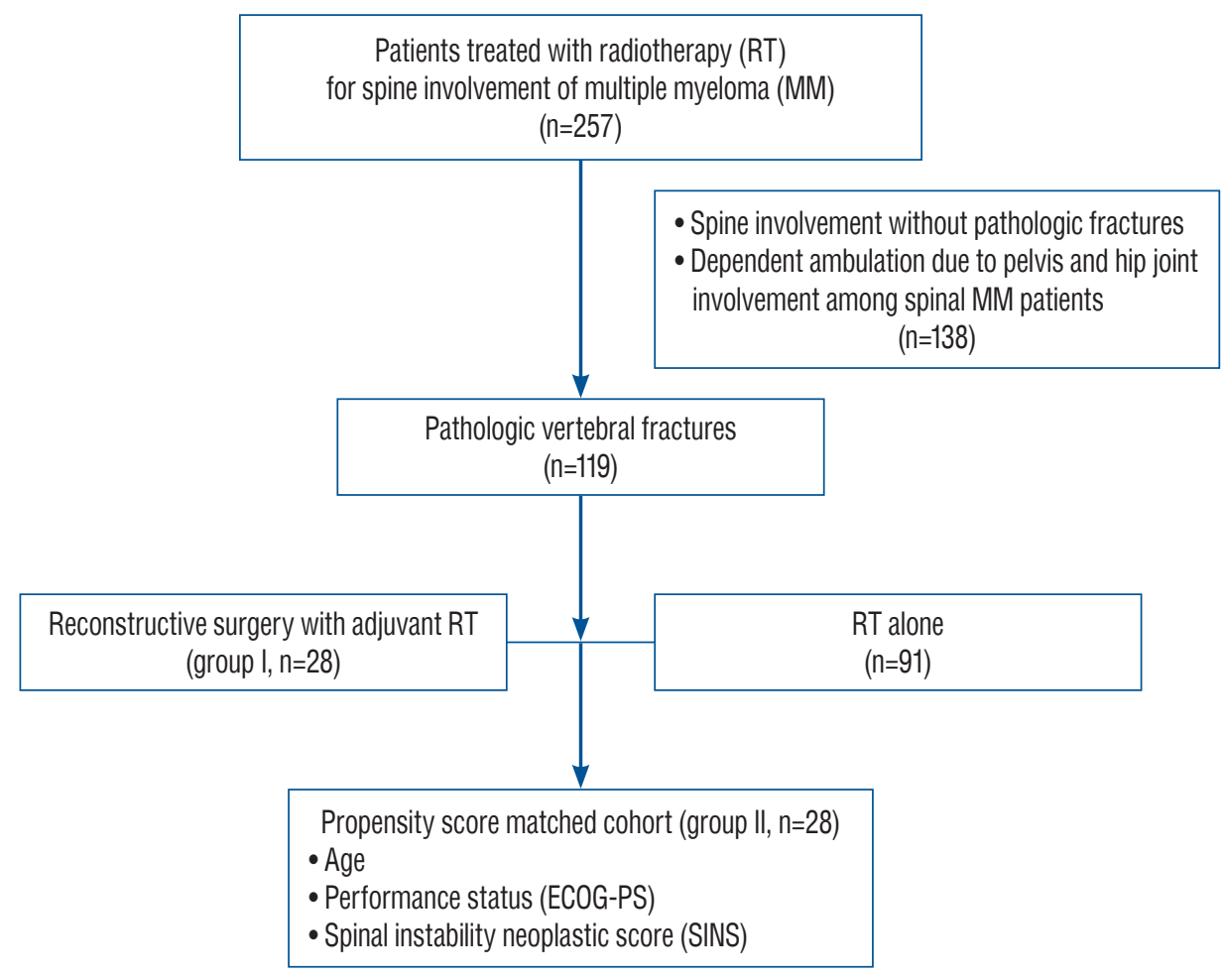

Fig. 1. Flow chart of patients included in the propensity score-matched cohort study. ECOG : Eastern Cooperative Oncology Group.

spinal column and neurological status, the basic strategy entailed posterior instrumented fusion with decompression and an additional anterior support was used in patients with severe vertebral body collapse.

\section{Radiological, neurological and clinical param- eters}

Clinical features of MM including the time from diagnosis to treatment, the International Staging System for MM (ISS), and PBSCT at the time of treatment of PVFs were recorded ${ }^{23)}$. Overall survival and independent risk factors for survival were evaluated. Symptoms correlating with the radiological measurements were pooled and analyzed in cases involving multiple vertebral segments. PVFs were confirmed radiologically by both radiologists and orthopedic surgeons ${ }^{19)}$. Radiological measurements included elements of instability on plain radiographs and CT, and paravertebral mass formation, epidural spinal cord compression (ESCC) on MRI ${ }^{7,11}$.

Neurological status including Frankel grade at baseline and last follow-up, and duration of independence ambulation, was assessed. Patients who were able to walk two or more steps with or without the use of an assistive device and less than grade 3 in ECOG-PS were considered independent ambulato$\mathrm{ry}^{26)}$. Bowel dysfunction was associated with constipation, loss of sphincter control, whereas urinary retention was recorded as bladder dysfunction. Clinical outcomes included performance status such as ECOG-PS and Karnofsky performance score (KPS), and numeric rating scale (NRS) for back pain at baseline and last follow-up. Treatment-related complications were also assessed along with surgical intervention and RT. Radiological and clinical parameters were assessed before and after the treatment. Patients had regular follow-up assessments every $2-3$ months until the end of the study or death.

\section{Statistical analyses}

Continuous variables, presented as the means and standard deviations, were compared using the Student's t-test or the Wilcoxon rank sum test. Categorical variables were compared using Fisher's exact test or Pearson's chi-square test, depending on the sample size. The variable with $p<0.10$ in the univariate analysis was included in multivariate analysis using logistic regression test. Cumulative survival rate and median survival of independent ambulation were analyzed using Kaplan-Meier survivorship analysis with log-rank test. Statistical 
analyses were carried out using the SPSS software (IBM SPSS Statistics for Windows, version 24.0; IBM Corp., Armonk, NY, USA) with a statistical significance of 0.05 .

\section{RESULTS}

\section{Patient demographics}

Clinical presentations and radiological findings of a total of 56 study patients at the time of treatment of PVFs are summarized in Table 1. Although multiple level involvement was significantly lower in group I than in group II (46.4\% vs. $78.6 \%$, $p=0.013$ ), other parameters were similar in both groups. Limit of motion (76.8\%) was the most common clinical presentation followed by axial pain (55.3\%) and neurologic deficit (53.6\%). Multiple level involvement, paravertebral mass, and ESCC grade $>1$ were found in more than $60 \%$ of patients.

Patient demographics and clinical features of the two study groups are shown in Table 2. Before the treatment, baseline disease characteristics including ISS stage, and rate of PBSCT, chemotherapy and mean radiation dose were similar. Thoracic and thoracolumbar junctions were most frequently involved in both groups. Mean SINS was not significantly different between the two groups (group I, 11.0 \pm 1.8 ; group II, 10.1 \pm 1.9 ), suggesting potential instability of the spinal column in both groups because total SINS scores of 7 to 12 are considered potentially unstable ${ }^{11)}$. Twenty patients underwent decompression with posterior instrumentation. Two patients underwent reconstruction with plate fixation after anterior corpectomy and six patients underwent combined anterior and posterior interventions via anterior corpectomy with mesh-type cages and posterior decompression with instrumentation (Fig. 2). Seven patients treated with percutaneous vertebroplasty in group II were excluded after propensity score matching.

\section{Neurological and clinical outcomes}

The median survival time of all patients was 46.1 months. Patients in group I survived 69.0 months compared with 32.7 months in group II without statistical significance $(p=0.146)$. In multivariate analysis, the only independent factor affecting survival was ECOG-PS $(>3)$ at the last follow-up (odds ratio [OR], 9.8; 95\% confidence interval [CI], 4.1-23.6; $p<0.01$ ). Mean duration of independent ambulation was also longer in group I (88.8 months; 95\% CI, 66.0-111.5) than in group II

Table 1. Clinical presentations and radiologic findings of multiple myeloma at the time of treatment for pathologic vertebral fracture $(n=56)$

\begin{tabular}{|c|c|c|c|c|}
\hline Variable & $\begin{array}{c}\text { Group I } \\
\text { (surgery+RT, } n=28)\end{array}$ & $\begin{array}{c}\text { Group II } \\
\text { (RT alone, } n=28 \text { ) }\end{array}$ & $p$-value & Total patients \\
\hline \multicolumn{5}{|l|}{ Clinical presentation } \\
\hline Limit of motion & $23(82.1)$ & $20(71.4)$ & 0.342 & $43(76.8)$ \\
\hline Axial pain & $16(57.1)$ & $15(53.6)$ & 0.788 & $31(55.3)$ \\
\hline Neurologic deficit & $18(64.3)$ & $12(42.9)$ & 0.108 & $30(53.6)$ \\
\hline Radiculopathy & $9(32.1)$ & $6(21.4)$ & 0.365 & $15(26.8)$ \\
\hline \multicolumn{5}{|l|}{ Radiologic finding } \\
\hline Multiple level involvement & $13(46.4)$ & $22(78.6)$ & 0.013 & $35(62.5)$ \\
\hline Paravertebral mass & $19(67.9)$ & $15(53.6)$ & 0.274 & $34(60.7)$ \\
\hline De novo deformity (kyphosis) & $18(64.3)$ & $12(42.9)$ & 0.108 & $30(53.6)$ \\
\hline Epidural spinal cord compression & & & 0.758 & \\
\hline 0 & $4(14.3)$ & $4(14.3)$ & & $8(14.3)$ \\
\hline 1a & $1(3.6)$ & $3(10.7)$ & & $4(7.2)$ \\
\hline $1 b$ & $3(10.7)$ & $3(10.7)$ & & $6(10.7)$ \\
\hline 1c & $3(10.7)$ & $2(7.1)$ & & $5(8.9)$ \\
\hline 2 & $11(39.3)$ & $9(32.1)$ & & $20(35.7)$ \\
\hline 3 & $6(21.4)$ & $7(25.0)$ & & $13(23.2)$ \\
\hline
\end{tabular}

Values are presented as number (\%). RT : radiotherapy 
Table 2. Patient demographics and clinical features of multiple myeloma with pathologic vertebral fractures

\begin{tabular}{|c|c|c|c|}
\hline Variable & Group I (surgery+RT, n=28) & Group II (RT alone, $\mathrm{n}=28$ ) & $p$-value \\
\hline Age & $60.2 \pm 7.2$ & $61.2 \pm 10.1$ & 0.682 \\
\hline Gender, $\mathrm{M}: \mathrm{F}$ & $16: 12$ & $19: 09$ & 0.584 \\
\hline Time from diagnosis to treatment & $10.4 \pm 17.7$ & $18.4 \pm 28.0$ & 0.206 \\
\hline Newly diagnosed MM & $20(71.4)$ & $15(53.6)$ & 0.168 \\
\hline Relapsed MM & $8(28.6)$ & $13(46.4)$ & 0.168 \\
\hline ISS stages & & & 0.379 \\
\hline 1 & $10(35.7)$ & $7(25.0)$ & \\
\hline 2 & $10(35.7)$ & 8 (28.6) & \\
\hline 3 & $8(28.6)$ & $13(46.4)$ & \\
\hline PBSCT & $11(39.3)$ & $12(42.9)$ & 0.786 \\
\hline CTX before diagnosis of PVF & $11(39.3)$ & $16(57.1)$ & 0.285 \\
\hline CTX after diagnosis of PVF & $24(85.7)$ & $26(92.9)$ & 0.699 \\
\hline Radiation dose (Gy) & $21.7 \pm 7.5$ & $24.4 \pm 7.3$ & 0.183 \\
\hline Involved level & & & 1.000 \\
\hline Cervical & $2(7.1)$ & $2(7.1)$ & \\
\hline C-T junction & $3(10.7)$ & $3(10.7)$ & \\
\hline Thoracic & $11(39.3)$ & $14(50.0)$ & \\
\hline T-L junction & $11(39.3)$ & $5(17.9)$ & \\
\hline Lumbar & $1(3.6)$ & $4(14.3)$ & \\
\hline SINS & $11.0 \pm 1.8$ & $10.1 \pm 1.9$ & 0.143 \\
\hline Type of surgery & & & - \\
\hline Posterior only & $20(71.4)$ & & \\
\hline Anterior only & $2(7.1)$ & & \\
\hline Anterior-posterior combined & $6(21.5)$ & & \\
\hline Vertebroplasty & & $0(0.0)$ & \\
\hline
\end{tabular}

Values are presented as mean \pm standard deviation or number (\%) unless otherwise indicated. RT : radiotherapy, M : male, F : female, MM : multiple myeloma, ISS : The International Staging System for multiple myeloma, PBSCT : peripheral blood stem cell transplantation, CTX : chemotherapy, PVF : pathologic vertebral fracture, C-T : cervico-thoracic, T-L : thoracolumbar, SINS : spine instability neoplastic score
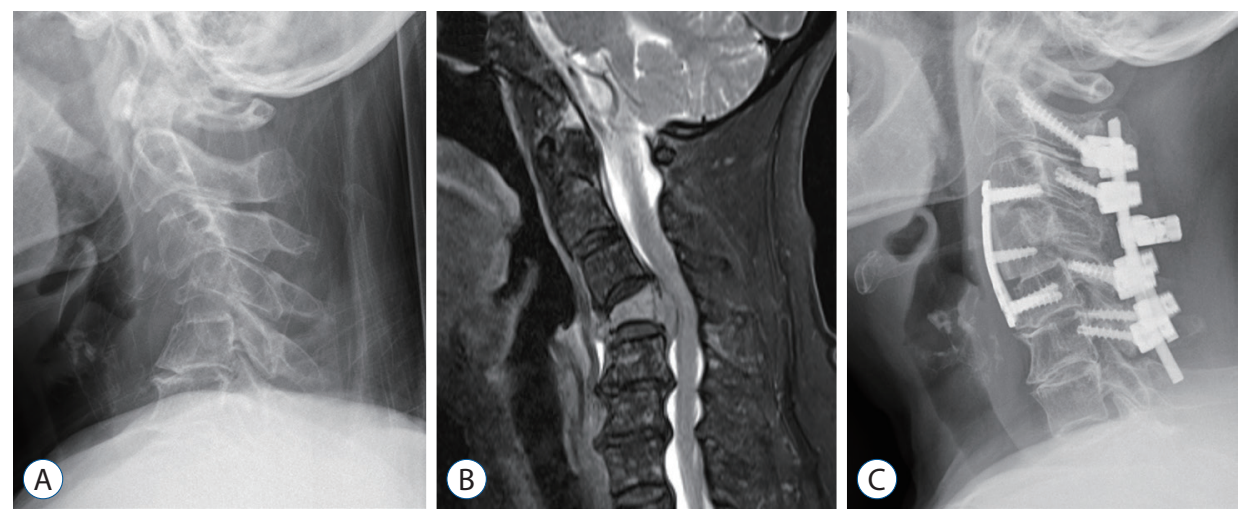

Fig. 2. A 72-year-old female patient undergoing reconstructive surgery for pathologic vertebral fracture due to multiple myeloma. A : Preoperative radiograph and (B) magnetic resonance imaging reveal a C4 pathologic fracture (de novo kyphosis) with epidural extension. C : Postoperative radiograph revealing anterior corpectomy with plate fixation and laminectomy with posterior screw fixation. 
(39.4 months; 95\% CI, 25.2-53.6) (log rank test; $p=0.022$ ) (Fig. 3). Similar neurological status was found in 15 patients belonging to group I and 14 patients in group II remained. Although both group manifested similar improvement of Frankel grade, the rate of deterioration in Frankel grade was significantly lower in group I than in group II (8.1\% vs. $28.6 \%$, $p=0.036$ ). Moreover, bowel/bladder dysfunction at the last follow-up was significantly lower in group I than in group II (17.9\% vs. $46.4 \%, p=0.022$ ) (Table 3 ).

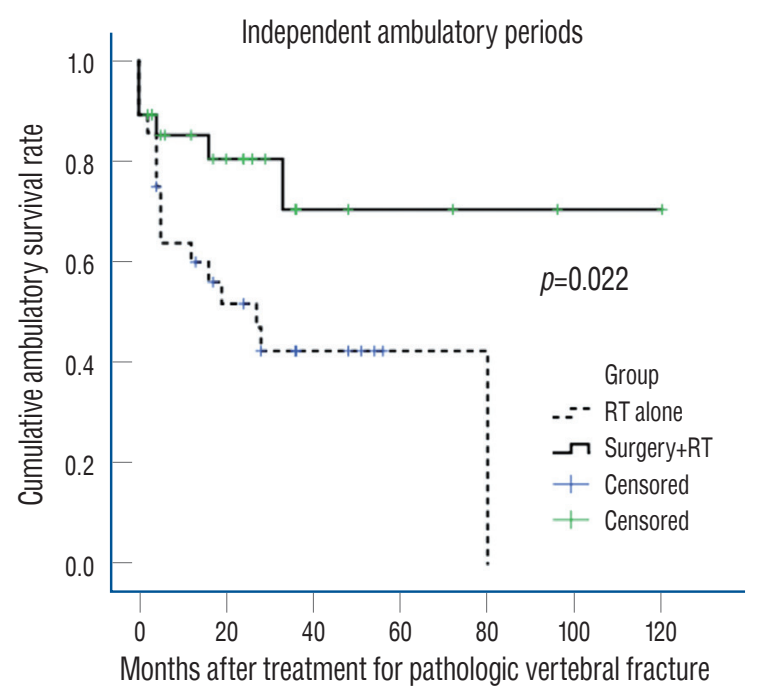

Fig. 3. Cumulative rates of independent ambulation in the two groups. $\mathrm{RT}$ : radiotherapy.
Although initial ECOG-PS and KPS values were similar in both groups, KPS at last follow-up was significantly greater in group I than in group II $(74.3 \% \pm 16.4 \%$ vs. $60.0 \% \pm 24.0 \%$, $p=0.028)$. In addition, NRS for back pain at last follow-up was lower in group I than in group II $(2.7 \pm 2.1$ vs. $5.0 \pm 2.2$, $p=0.0001)$ despite similar initial NRS. The rate of treatmentrelated complications was similar in both groups. In group I, four patients suffered from surgery-related wound complications (including wound infection in two cases and wound dehiscence and seroma in one case). In group II, three patients had RT-related complications including pleural effusion in two patients and tumor lysis syndrome in one patient (Table 4).

\section{DISCUSSION}

MM cells stimulate osteoclastogenesis and inhibit osteoblastogenesis in areas adjacent to tumor leading to osteolytic bone resorption ${ }^{5,6,28,30,32)}$. With increasing survival of MM patients due to advances in medical treatments, the prevalence of morbidity and mortality due to musculoskeletal injury will be critical in the treatment of $\mathrm{MM}^{5,14,28,30)}$. Especially, it is essential to effectively manage MM with spinal involvement in order to prevent permanent disability and improve quality of life (QOL) during treatment ${ }^{14,18)}$.

Because of its radio-sensitivity, RT also plays an important

Table 3. Changes of neurologic status between the two groups

\begin{tabular}{|c|c|c|c|c|c|c|c|c|c|c|c|}
\hline \multirow{3}{*}{ Parameter } & \multicolumn{5}{|c|}{ Group I (surgery+RT, n=28) } & \multicolumn{5}{|c|}{ Group II (RT alone, $n=28$ ) } & \multirow{3}{*}{$p$-value } \\
\hline & \multicolumn{5}{|c|}{ Frankel grade (last) } & \multicolumn{5}{|c|}{ Frankel grade (last) } & \\
\hline & A & B & $\mathrm{C}$ & D & $\mathrm{E}$ & A & B & $\mathrm{C}$ & D & E & \\
\hline \multicolumn{12}{|l|}{ Frankel grade (Initial) } \\
\hline A & 1 & 1 & 1 & & & & & & & & \\
\hline B & & & & 1 & & & 1 & 1 & & & \\
\hline C & & & & 2 & & & & 1 & & & \\
\hline D & & & & 6 & 6 & & 2 & & 2 & 5 & \\
\hline$E$ & & & & 2 & 8 & & & 2 & 4 & 10 & \\
\hline $\begin{array}{l}\text { Mean duration of independent ambulation } \\
\text { (months) }\end{array}$ & \multicolumn{5}{|c|}{$88.8(66.0-111.5)$} & \multicolumn{5}{|c|}{$39.4(25.2-53.6)$} & 0.022 \\
\hline Improvement of Frankel grade & \multicolumn{5}{|c|}{$11(39.3)$} & \multicolumn{5}{|c|}{$6(21.4)$} & 0.146 \\
\hline Deterioration of Frankel grade & \multicolumn{5}{|c|}{$2(8.1)$} & \multicolumn{5}{|c|}{$8(28.6)$} & 0.036 \\
\hline Bowel/bladder dysfunction & \multicolumn{5}{|c|}{$5(17.9)$} & \multicolumn{5}{|c|}{$13(46.4)$} & 0.022 \\
\hline
\end{tabular}

Values are presented as mean (95\% confidence interval) or number (\%). RT : radiotherapy 
role in palliation of symptoms and prophylaxis against local recurrence during the treatment of spinal $\mathrm{MM}^{20-22}$. However, surgical intervention is recommended for patients with spinal instability and neurological deficits due to compressive lesion $^{8,33,34)}$. Clinical efficacies of reconstructive surgery combined with RT for spinal MM have yet to be established. The aim of this study was to compare the clinical efficacies of reconstructive surgery with RT and RT alone, focusing on PVFs and in similar cohorts based on propensity score matching despite retrospective comparative study.

The most common clinical presentation was attributed to mechanical instability such as limitation of motion (76.8\%) and axial pain (55.3\%) during treatment for PVFs. Combined neurologic deficits and radiculopathy were found in 53.6\% and $26.8 \%$ of patients, respectively. Radiological findings revealed both spinal deformity such as kyphosis (53.6\%) and cord compression including ESCC grade 2 or 3 (58.9\%). The PVFs in spinal MM was characterized by both mechanical instability and neural compression.

In this study, patients in group I survived longer than 36.3 months compared with those in group II, but the difference was not statistically significant (69.0 vs. 32.7 months, $p=0.146$ ) The only independent factor affecting survival was good performance status at the last follow-up. Several studies have reported that surgical intervention played an adjunct role in preventing gross spinal deformity or neurologic deficits, without improving survival rate in the treatment of $\mathrm{MM}^{2,4,13,18)}$. However, recent studies emphasized the effect of surgical interventions on functional status and disease prognosis as well as survival, although the effect on improved survival has yet to be established ${ }^{3,27,31,34)}$. Therefore, further studies are needed to evaluate the effect of surgery on survival, considering the small number of included cohorts and the low value of $p$-value in this study.

The mean duration of independent ambulation was also longer in group I than in group II (88.8 vs. 39.4 months, $p=0.022$ ). Further, the deterioration of Frankel grade and bowel/bladder dysfunction were significantly lower in group I than in group II ( $8.1 \%$ vs. $28.6 \%$ for deterioration; $17.9 \%$ vs. $46.4 \%$ for dysfunction). Direct decompression in surgical intervention might be related to better neurological function compared with RT alone. In the case of epidural mass causing neurologic deficit, it is difficult to administer high levels of RT

Table 4. Clinical outcomes including treatment-related complications between the two groups

\begin{tabular}{lccc}
\hline Variable & Group I (surgery+RT, $\mathbf{n}=\mathbf{2 8})$ & Group II (RT alone, $\mathbf{n}=\mathbf{2 8})$ & $p$-value \\
\hline Initial ECOG-PS & & & \\
$0-2$ & $22(78.6)$ & $24(85.7)$ & 0.485 \\
$3-4$ & $6(21.4)$ & $4(14.3)$ & \\
Last ECOG-PS & & & \\
$0-2$ & $23(82.1)$ & $18(64.3)$ & 0.131 \\
$3-4$ & $5(17.9)$ & $10(35.7)$ & 0.162 \\
Initial KPS & $73.2 \pm 17.0$ & $79.6 \pm 16.9$ & 0.028 \\
Last KPS & $74.3 \pm 16.4$ & $60.0 \pm 24.0$ & 0.556 \\
Initial NRS (back) & $6.4 \pm 1.3$ & $6.0 \pm 2.3$ & 0.0001 \\
Last NRS (back) & $2.7 \pm 2.1$ & $5.0 \pm 2.2$ & 1.000 \\
Treatment-related complications & & & \\
Pleural effusion & $0(0.0)$ & $2(7.1)$ & \\
Tumor lysis syndrome & $0(0.0)$ & $1(3.6)$ & \\
Wound infection & $2(7.1)$ & $0(0.0)$ & \\
Wound dehiscence & $1(3.6)$ & $0(0.0)$ & \\
Wound seroma & $1(3.6)$ & $3(10.7)$ & \\
Total & $4(14.3)$ & & \\
\hline
\end{tabular}

Values are presented as mean \pm standard deviation or number (\%). RT : radiotherapy, ECOG-PS : Eastern Cooperative Oncology Group-performance status, KPS : Karnofsky performance score, NRS : numeric rating scale 
because of the spinal cord nearby. In this regard, local recurrence and nerve compression have been reported to be not easily controlled by RT alone ${ }^{211}$.

Unstable spine due to PVFs might also be associated with long-term outcomes ${ }^{15,24)}$. SINS in both groups suggested potentially unstable spine, and all patients in group I and matched cohorts in group II manifested potentially unstable or unstable lesions ${ }^{11}$. Several studies have recommended the use of vertebroplasty or kyphoplasty for osteolytic lesion ${ }^{2,4,13,311}$. However, in case of obvious mechanical instability, reconstructive surgery should be considered to preserve long-term mechanical stability and neural decompression ${ }^{11,15)}$. In our study, seven patients treated with vertebroplasty in group II were not included after propensity score matching based on SINS. Therefore, the stability of the spinal column prevents the development of kyphosis with subsequent cord compression, and result in superior outcomes in patients who underwent reconstructive surgery. Furthermore, adjuvant RT is also important to eradicate the residual MM cells and reduce the local recurrence after the surgery ${ }^{31}$.

Although initial NRS values for back pain were similar in both groups, NRS at last follow-up was significantly lower in group I than in group II (2.7 vs. 5.0 points), which was consistent with a previous report suggesting that RT might not be useful in controlling pain, because MM is usually related to structural damage during the majority of the time ${ }^{18)}$. Similarly, treatment of spine with could negatively impact bone marrow function, and does not prevent progressive osteolytic collapse or alleviate concurrent back pain ${ }^{29)}$. Treatment-related complications were similar in both groups. Interestingly, four patients in group I reported surgery-related wound complications and three patients in group II had RT-related complications. Surgery-related complications were resolved with wound care under local anesthesia and intravenous antibiotics. RT-related complications were also resolved via medical treatments, including diuretics and pigtail catheter insertion for pleural effusion and hydration for tumor lysis syndrome.

This study has some limitations. First, the multidisciplinary approach based on surgical treatment via reconstructive surgery for PVFs in MM was not based on validated and uniform surgical indication. Therefore, we designed the propensity score matched study to ensure similar baseline characteristics such as age and ECOG-PS, and spinal instability as SINS to minimize the selection bias. Moreover, medical treatments in- cluding chemotherapy regimens and RT protocols were completely same in both groups. Second, this is a retrospective study with relatively small number of cohorts due to the rarity of reconstructive surgery for spinal MM. The heterogeneity of spine levels and surgical approaches used is also a limitation. Third, a longitudinal evaluation of radiological findings was not conducted in this study. Because progressive mechanical instability and neural compression is a key pathological mechanism for PVFs in MM, a prospective study with longitudinal evaluation would have been helpful in delineating the mechanism. Finally, spine stereotactic radiosurgery has been reported to deliver highly conformal radiation effectively to MM while minimizing the dose to the spinal cord compared to conventional $\mathrm{RT}^{22)}$. However, the impact of radiosurgery on spinal MM was not evaluated in this study. In the near future, multi-center, prospective studies with an adequate number of cohorts are needed to elucidate the appropriate treatment options depending on the type of surgery, including radiosurgery with minimally invasive surgery such as percutaneous fixation or vertebroplasty ${ }^{25)}$.

Despite these limitations, to the best of our knowledge, this study is the first matched cohort study comparing reconstructive surgery with RT and RT alone for PVFs from spinal MM. The primary treatment goal for metastatic spine tumors is maintenance or improvement of $\mathrm{QOL}^{1}$. Therefore, this study demonstrated clear advantages in maintaining spinal stability and neurologic function in reconstructive surgery compared with RT alone for unstable PVFs. These findings could facilitate decision-making by physicians and surgeons considering reconstructive surgery for patients with spinal MM.

\section{CONCLUSION}

This study revealed that survival rates of MM patients with PVFs were similar between the reconstructive surgery with adjunctive RT and RT alone. However, reconstructive surgery ensured maintenance of independent ambulation and neurological status, and pain control compared with RT alone with similar complications. Therefore, reconstructive surgery with RT for unstable PVFs due to spinal MM is a considerable option for patients with better performance status and longer life expectancy in order to maintain QOL, namely "walking and living their life". 


\section{CONFLICTS OF INTEREST}

No potential conflict of interest relevant to this article was reported.

\section{INFORMED CONSENT}

This type of study does not require informed consent.

\section{AUTHOR CONTRIBUTIONS}

\author{
Conceptualization : YHK \\ Data curation : JHA, SIK, JWJ \\ Formal analysis : HYP, JHA \\ Funding acquisition : YHK \\ Methodology : HYP, YHK, SIK \\ Project administration : YHK, KYH \\ Visualization : JHA, JWJ \\ Writing - original draft : HYP, JHA \\ Writing - review \& editing: YHK, KYH
}

\section{ORCID}

Hyung-Youl Park https://orcid.org/0000-0002-0084-8867

Young-Hoon Kim https://orcid.org/0000-0003-1237-4600

Joo-Hyun Ahn https://orcid.org/0000-0003-4752-6213

Kee-Yong $\mathrm{Ha} \quad$ https://orcid.org/0000-0002-4059-7943

Sang-Il Kim https://orcid.org/0000-0002-2758-0012

Jae-Woong Jung https://orcid.org/0000-0002-7817-2422

\section{- Acknowledgements}

This work was supported by the National Research Foundation of Korea (NRF) grant funded by the Korea government (MSIT) (No. 52019A015400438).

\section{References}

1. Abrahm JL, Banffy MB, Harris MB : Spinal cord compression in patients with advanced metastatic cancer: "all I care about is walking and living my life". JAMA 299 : 937-946, 2008

2. Amelot A, Cristini J, Salaud C, Moles A, Hamel O, Moreau P, et al. : Overall survival in spine myeloma metastases: difficulties in predicting with prognostic scores. Spine (Phila Pa 1976) 42 : 400-406, 2017

3. Amelot A, Moles A, Cristini J, Salaud C, Touzeau C, Hamel O, et al. : Predictors of survival in patients with surgical spine multiple myeloma metastases. Surg Oncol 25 : 178-183, 2016

4. Anselmetti GC, Manca A, Montemurro F, Hirsch J, Chiara G, Grignani $\mathrm{G}$, et al. : Percutaneous vertebroplasty in multiple myeloma: prospective long-term follow-up in 106 consecutive patients. Cardiovasc Intervent Radiol 35 : 139-145, 2012

5. Barillé-Nion $S$, Bataille R : New insights in myeloma-induced osteolysis. Leuk Lymphoma 44 : 1463-1467, 2003

6. Bataille R, Chappard D, Marcelli C, Dessauw P, Baldet P, Sany J, et al. : Recruitment of new osteoblasts and osteoclasts is the earliest critical event in the pathogenesis of human multiple myeloma. J Clin Invest $88: 62-66,1991$

7. Bilsky MH, Laufer I, Fourney DR, Groff M, Schmidt MH, Varga PP, et al. : Reliability analysis of the epidural spinal cord compression scale. J Neurosurg Spine 13 : 324-328, 2010

8. Chang SY, Mok S, Park SC, Kim H, Chang BS : Treatment strategy for metastatic spinal tumors: a narrative review. Asian Spine J 14 : 513525,2020

9. Cho YK, Kim SI, Ha KY, Kim YH, Park HY, Min CK : Increased osteoblastic activity suppressed proliferation of multiple myeloma plasma cells. Spine (Phila Pa 1976) 44 : E384-E392, 2019

10. Fisher CG, DiPaola CP, Ryken TC, Bilsky MH, Shaffrey $\mathrm{Cl}$, Berven $\mathrm{SH}$, et al. : A novel classification system for spinal instability in neoplastic disease: an evidence-based approach and expert consensus from the Spine Oncology Study Group. Spine (Phila Pa 1976) 35 : E1221-E1229, 2010

11. Fourney DR, Frangou EM, Ryken TC, Dipaola CP, Shaffrey $\mathrm{Cl}$, Berven $\mathrm{SH}$, et al. : Spinal instability neoplastic score: an analysis of reliability and validity from the spine oncology study group. J Clin Oncol 29 : 30723077, 2011

12. Greipp PR, San Miguel J, Durie BG, Crowley JJ, Barlogie B, Bladé J, et al. : International staging system for multiple myeloma. J Clin Oncol 23 : 3412-3420, 2005

13. Ha KY, Min CK, Seo JY, Kim YH, Ahn JH, Hyun NM, et al. : Bone cement augmentation procedures for spinal pathologic fractures by multiple myeloma. J Korean Med Sci 30 : 88-94, 2015

14. Kim SI, Kim YH, Ha KY, Lee JW, Lee JW : Surgical roles for spinal involvement of hematological malignancies. J Korean Neurosurg Soc 60 : 534-539, 2017

15. Kim YH, Kim J, Chang SY, Kim H, Chang BS : Treatment strategy for impending instability in spinal metastases. Clin Orthop Surg 12 : 337342,2020

16. Kyle RA : Multiple myeloma: review of 869 cases. Mayo Clin Proc 50 : 29-40, 1975

17. Kyle RA, Gertz MA, Witzig TE, Lust JA, Lacy MQ, Dispenzieri A, et al. : Review of 1027 patients with newly diagnosed multiple myeloma. Mayo Clin Proc 78 : 21-33, 2003 
18. Latif $\mathrm{T}$, Hussein MA : Advances in multiple myeloma and spine disease. Clin Lymphoma Myeloma 6 : 228-233, 2005

19. Lecouvet FE, Vande Berg BC, Maldague BE, Michaux L, Laterre E, Michaux JL, et al. : Vertebral compression fractures in multiple myeloma. Part I. Distribution and appearance at MR imaging. Radiology 204 : 195199, 1997

20. Mikhael JR, Dingli D, Roy V, Reeder CB, Buadi FK, Hayman SR, et al. : Management of newly diagnosed symptomatic multiple myeloma: updated Mayo Stratification of Myeloma and Risk-Adapted Therapy (mSMART) consensus guidelines 2013. Mayo Clin Proc 88 : 360-376, 2013

21. Mill WB : Radiation therapy in multiple myeloma. Radiology $115: 175-$ 178, 1975

22. Miller JA, Balagamwala EH, Chao ST, Emch T, Suh JH, Djemil T, et al. : Spine stereotactic radiosurgery for the treatment of multiple myeloma. J Neurosurg Spine 26 : 282-290, 2017

23. Palumbo A, Avet-Loiseau H, Oliva S, Lokhorst HM, Goldschmidt H, Rosinol L, et al. : Revised international staging system for multiple myeloma: a report from International Myeloma Working Group. J Clin Oncol 33 : 2863-2869, 2015

24. Park HY, Ahn JH, Ha KY, Kim YH, Kim SI, Min HK, et al. : Clinical and radiologic features of osteoporotic spine fracture with delayed neurologic compromises. World Neurosurg 120 : e1295-e1300, 2018

25. Park J, Ham DW, Kwon BT, Park SM, Kim HJ, Yeom JS : Minimally invasive spine surgery: techniques, technologies, and indications. Asian Spine J $14: 694-701,2020$

26. Patchell RA, Tibbs PA, Regine WF, Payne R, Saris S, Kryscio RJ, et al. : Direct decompressive surgical resection in the treatment of spinal cord compression caused by metastatic cancer: a randomised trial. Lancet $366: 643-648,2005$

27. Qian J, Jing J, Tian D, Yang H : Partial tumor resection combined with chemotherapy for multiple myeloma spinal cord compression. Ann Surg Oncol 21 : 3661-3667, 2014

28. Roodman GD : Pathogenesis of myeloma bone disease. Leukemia 23 : 435-441, 2009

29. Rosen LS, Gordon D, Kaminski M, Howell A, Belch A, Mackey J, et al. : Zoledronic acid versus pamidronate in the treatment of skeletal metastases in patients with breast cancer or osteolytic lesions of multiple myeloma: a phase III, double-blind, comparative trial. Cancer J 7 : 377 387, 2001

30. Sezer 0 : Myeloma bone disease: recent advances in biology, diagnosis, and treatment. Oncologist $14: 276-283,2009$

31. Utzschneider $S$, Schmidt $H$, Weber $P$, Schmidt GP, Jansson V, Dürr HR : Surgical therapy of skeletal complications in multiple myeloma. Int Orthop 35 : 1209-1213, 2011

32. Yaccoby $S$ : Advances in the understanding of myeloma bone disease and tumour growth. Br J Haematol 149 : 311-321, 2010

33. Yoo SL, Kim YH, Park HY, Kim SI, Ha KY, Min HK, et al. : Clinical significance of preoperative embolization for non-hypervascular metastatic spine tumors. J Korean Neurosurg Soc 62 : 106-113, 2019

34. Zadnik PL, Goodwin CR, Karami KJ, Mehta Al, Amin AG, Groves ML, et al. : Outcomes following surgical intervention for impending and gross instability caused by multiple myeloma in the spinal column. J Neurosurg Spine $22:$ 301-309, 2015 